\title{
Industrial Parameter Monitoring and Controlling Using Gsm and Web Server
}

\author{
Mr. Jadhav sunny P, Mr. Gaikwad Siddesh R, Mr.Hase Kiran D, Mr. Mandlik \\ S. B.
}

\begin{abstract}
Remote Monitoring, and Control is one of the most important and necessary criteria for increasing production and process plant availability. There is lot of development in industry and the requirement for industrial monitoring system is getting higher. System should be able to acquire, save, analyze, and process real time data. It is also required controlling particular machines, to change related environment factors and monitoring in long distance so that it realizes modern, intelligent, and accurate control. We can achieve these advantages by the substitution of embedded ARM processor to realize data acquisition and control (DACS). This DACS system measures the remote signals and controls the remote devices through reliable protocols and communication network as a web server. In addition of that a GSM Mobile Communication will help to providing information about related parameter to the system when web server is not available to client.
\end{abstract}

\section{Introduction}

Data acquisition system is most important in industry and consumer applications. In many applications, human beings have been replaced by unmanned devices that will acquire data and relay the data back to the base. There are data-acquisition and control devices that will be a substitute for a supervisor in a multisite job operation.

Although these are well-built systems that serve the purpose for a specific task, the user cannot interact with the system, which uses the Global System for Mobile Communications (GSM) a popular wireless choice for connectivity between the data-acquisition units and clients. A web server provides access to the end device as per the request of client. In these system there is a central functional unit that host web pages. In these applications, data are uploading on a central server and are then served to the clients via the Internet. A person that needs to access any data must first access the server. When configured IP address entered, predesigned web page get displayed through which we can monitor and control particular device. However, there is still no effort to minimize the operational costs (including the costs to transfer a large amount of data). In addition, this system is based on an industrial PC, thus making it an expensive solution. Interaction with the embedded unit is also an important issue. In, an embedded PC card placed on the Internet allows limited interaction through commands sent through Transmission Control Protocol/IP (TCP/IP) and User Datagram Protocol.

\section{ARM CORE HARDWARE}

\section{Hardware Design}

There are three part of system. First is the Embedded web server, second is client and third one is sensor part .The remote input/output data acquisition and control system based on embedded ARM platform has high universality. Sensors are used for process monitoring and for process control.

Each I/O channel can select a variety of electrical and non electrical signals like current, voltage, resistance etc. This signal is taken into LPC2148 \& digitized using the inbuilt ADC. External memory is used to store this data, we can directly show this data on LCD display which is connected to port $0 \&$ the memory is act as a data base during Accessing web server mode \& GSM mode.



Fig.1-Remote Monitoring \& Control of Industrial Parameters through Embedded Web Server \& GSM Mobile 
There is internal SPI module in ARM processor, which directly supports the Ethernet service communication. Therefore the data has been stored and controlled by some other PCs or network Ethernet. Also ARM Processor has serial communication interface (Rx, Tx, GND) Which is used to transferring data to GSM Modem.

b) CLIENT-SERVER ARCHITECTURE

A web server is a system which hosts web pages and provides services for any requesting clients. A web server can be embedded in a device to provide remote access to the device from a web browser. The embedded system can be utilized to serve the embedded web documents, including static and dynamic information about industry machineries/systems to web browsers [2].

A Client can access the industry's web server through internet and LAN router. Digitally acquired data are uploaded on web server and store in web server's data base. Whenever the client wants to access data, it sends the request to server; this request is taken by the router, which is connected to the internet.

The web processes the request made and finally connects to the desired web server, access the requested data and sends the data to the client.

The concept of proposed system is shown on Fig.B, with embedded web server on a single chip module. This is a single hardware it contains RTOS portable ARM processor. ARM processor is heart of system which is the responsible part for measuring signals and controlling the devices automatically. Measurements can be done by DACS mode and the data are shared with clients thro embedded web server by embedded web server mode. All the tasks are manages by the real time operating system such as measuring signals, A/D conversion, data base updating,sendingHTMLpages and connecting/commu icating with new users etc [2].

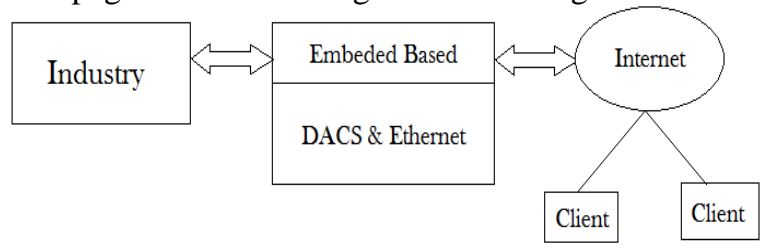

Fig. 2 - Embedded Web-Server architecture [2]

c)SPI (Serial Peripheral Interface) STANDERD

It has a 4-wire synchronous serial interface. Data communication is enabled with a low active Slave Select or Chip Select Signal (SS) or (CS). Data is transmitted with a 3- wire interface consisting of wires for serial data input (MOSI), serial data output (MISO) and serial clock (SCK) [3].

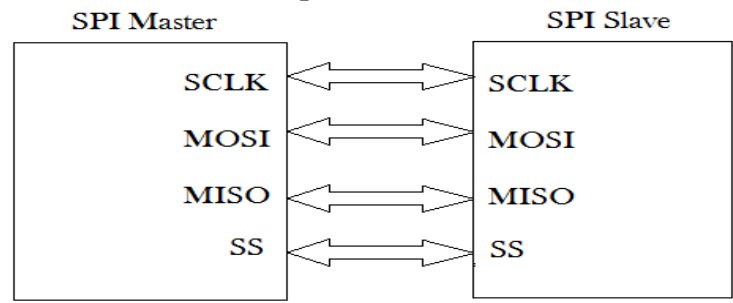

Fig.3-Serial Peripheral Interface[3]

\section{SPI Protocol}

The serial communication is performed by means of two pins that are SI and SO as shown in Fig.4. SCLK provides clock synchronization and CS is the chip select. This communication technique can be implemented between processor and peripherals that have SPI interface. Serial Peripheral Interface Bus is a synchronous serial data link standard where communication is performed in master/slave mode and master device initiates the data frame. This is a full duplex mode of point to point communication. The serial clock, SCLK generated by the master device is used by the slave also. The SS which is the Slave Select signal should in active low state for the slave to have communication with master. This is a four wire communication as shown in Fig. 4. The SDO or Serial Data Output signal send by the master and after receiving the clock pulse, the slave device responds back with SDI or Serial Data Input signal [4].

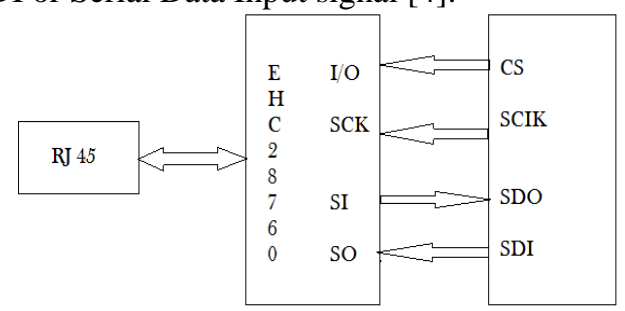

Fig.4-SPI Interface [4] 


\section{a) ETHERNET}

Ethernet is the family of wired network technology. And Ethernet is standardized as IEEE 802.3. The data of Ethernet is grouped into bytes often called frame. The start of the frame is preamble which is of 7 bytes length. It contains sets of 0's and 1's arranged alternately. The SFD or start frame delimiter is a 1 byte binary value. Its left most end contains a ' 11 ' by identifying it, the receiver gets information about arrival of the new frame. Then comes the 48-bit MAC address that contains both source and destination address. The type of length which is of 2 bytes gives information about the protocol bound in the succeeding data payload. The frame terminates with a 32-bit checksum that performs CRC checking to identify if any error is present [4].

\section{Gsm Modem}

A GSM modem is a specialized type of modem, which accepts a SIM card, and operates over a subscription to a mobile operator, just like a mobile phone. From the mobile operator perspective, a GSM modem looks just like a mobile phone [5]. A GSM modem can be a dedicated modem device with a serial, USB or Bluetooth connection, or it may be a mobile phone that provides GSM modem capabilities.

GSM/GPRS RS232 Modem from rhydo LABZ is built with SIMCOM Make SIM900 Quad-band GSM/GPRS engine, works on frequencies $850 \mathrm{MHz}, 900 \mathrm{MHz}, 1800 \mathrm{MHz}$ and $1900 \mathrm{MHz}$. It is very compact in size and easy to use as plug in GSM Modem [7]. The Modem is designed with RS232 Level converter circuitry, which allows you to directly interface PC Serial port .The baud rate can be configurable from 9600-115200 through AT command. Initially Modem is in Auto baud mode. This GSM/GPRS RS232 Modem is having internal TCP/IP stack to enable you to connect with internet via GPRS. It is suitable for SMS as well as DATA transfer application in M2M interface.

The modem needed only 3 wires (Tx, Rx, GND) except Power supply to interface with microcontroller/Host PC. The built in Low Dropout Linear voltage regulator allows you to connect wide range of unregulated power supply $(4.2 \mathrm{~V}-13 \mathrm{~V})$ to send \& Read SMS [7].

\section{a) MOBILE PHONE}

A mobile phone also known as a wireless phone, cell phone, or cellular telephone is a little portable radio telephone. Mobile Phone can serve as powerful tool for world-wide communication. The Mobile Phone is a natural choice, since it is a communication resource generally available by people, which makes them practically always contactable and capable to send commands to operate the parameters in the industries.

\section{Conclusion}

There are some application where constant temperature is needed so we have to declare set point. If temperature below the set point heater will be on and if it is above the set point cooler will be on.

To transmit data from existing device with serial peripheral interface to network, Ethernet monitoring \& controlling system which is based on web browser.

This system can be used mostly in remote data acquisition and control system in industry.

\section{References}

[1] Ali Ziya Alkar, Member, IEEE, and Mehmet Atif Karaca " An Internet- Based Interactive Embedded Data-Acquisition System for Real-Time Applications" IEEE TRANSACTIONS ON INSTRUMENTATION AND MEASUREMENT, VOL. 58, NO. 3, MARCH 2009.J. Clerk Maxwell, A Treatise on Electricity and Magnetism, 3rd ed., vol. 2. Oxford: Clarendon, 1892, pp.68-73.

[2] MANIVANNAN M \& KUMARESAN N. Anna University of Technology Coimbatore Coimbatore, Tamilnadu, India "EMBEDDED WEB SERVER \& GPRS BASED ADVANCED INDUSTRIAL AUTOMATION USING LINUX RTOS", Vol. 2(11), 2010, 6074-6081.

[3] Mr. Suyog A. Wani Prof. R.P.Chaudhari "Ethernet Enabled Digital 1/0 Control in Embedded Systems", 2012 International Conference on Computing, Electronics and Electrical Technologies [ICCEET] [4] Alen Rajan, Aby K. Thomas, Rejin Mathew "A Comparative Performance Analysis of ARM based Web Servers with Integrated and External Ethernet Interfaces for Industrial Applications", International Journal of Computer Applications (0975 - 8887) Volume 44- No.21, April 2012

[5] Dr.B.Ramamurthy, S.Bhargavi, Dr.R.ShashiKumar,” Development of a Low-Cost GSM SMS-Based Humidity Remote Monitoring and Control system for Industrial Applications". (IJACSA) International Journal of Advanced Computer Science and Applications, Vol. 1, No. 4, October 2010.

[6] Manivannan M Kumaresan N "Design of On-line Interactive Data Acquisition and Control System for Embedded Real Time Applications" 978-1-4244-7926-9/11/\$26.00 (C2011 IEEE.

[7] SIM 900 -RS232 GSM/GPRS Modem User Manual. http://www.rhydolabz.com.

[8] Mr. Suyog A. Wani \& Prof. R.P.Chaudhari "Ethernet Enabled Digital i/o Control in Embedded Systems" 978-1-4673-02104112/\$31.00 (C2012 IEEE.

[9] M Poongothai “ARM Embedded Web Server Based on DAC System” 978-1-61284-764-1/11/\$26.00 (C2011 IEEE. 